\title{
HAPLOLOGIA SINTÁTICA E EFEITOS DE ECONOMIA
}

\author{
- Elisa Battisti -
}

RESUMO: Syntactic haplology (SH) is a sandhi rule which eliminates one of two contiguous identical or partially identical syllables in a sentence (caldo de cana $>$ cal de cana). Interpreting the results of a variable rule analysis of SH on southern Brazilian Portuguese data according to Optimality Theory (PRINCE and SMOLENSKY, 1993; MCCARTHY and PRINCE, 1993,1995) implied discussing the nature of SH, if coalescence or deletion, and the markedness restriction involved. SH can be interpreted as a deletion process motivated by the requirement of avoiding sequences of identical or partially identical syllabes, which is expressed by OCP.

PALAVRAS-CHAVE: Haplologia Sintática. Coalescência. Apagamento. OCP.

Haplologia é a denominação que se dá à omissão de alguns dos sons numa seqüência de articulações semelhantes (CRYSTAL, 2000, p.137). Ela é morfológica quando uma de duas sílabas iguais contíguas é suprimida na composição ou derivação (CÂMARA JR., 1984, p.134), como em tragicômico, de trágico + cômico, semínima, de semi + mínima; sintática quando omite-se uma de duas sílabas iguais ou semelhantes contíguas na frase (Silveira, 1971, p.79), como em caldo de cana $>$ cal de cana, Rio Grande do Sul> Rio Gran do Sul.

A discussão a ser aqui apresentada ${ }^{1}$, pautada pela Teoria da Otimidade (PRINCE e SMOLENSKY, 1993; MCCARTHY e PRINCE, 1993,1995), diz respeito à haplologia sintática, e relaciona-se a uma análise de regra variável em dados do português do sul do Brasil ${ }^{2}$. Interpretar os resultados dessa análise implica conceber o fenômeno com base em teoria fonológica. Em uma abordagem baseada em restrições e orientada pelo output como a Teoria da Otimidade (TO), pode-se conceber a não-realização de material fonológico tanto como apagamento, quanto como coalescência ${ }^{3}$. As restrições e sua interação é que darão origem a um ou outro processo. Questionase, então, a natureza da haplologia sintática no português brasileiro, se apagamento ou coalescência: que demandas interagem de modo a originar haplologia sintática?

Neste trabalho, revisam-se concepções de haplologia morfológica (HM) de que se podem derivar considerações sobre haplologia sintática (HS): a proposta de De Lacy (1999), de HM como coalescência, e a hipótese de Gouskova (2003) para processos de síncope, entre eles a HM, ambas na perspectiva da Teoria da Otimidade. Inicia-se o trabalho com o breve relato de resultados já obtidos na análise de regra variável.

\section{A REGRA VARIÁVEL DE HAPLOLOGIA SINTÁTICA}

A análise da HS como regra variável do português brasileiro falado no sul do país realizouse a partir de 24 entrevistas sociolingüísticas do corpus VARSUL (UFRGS, PUCRS, UFSC, UFPR), de informantes de Porto Alegre com Nível Superior de escolaridade. A variável dependente foi a haplologia sintática em seqüência de sílabas com / t/ e /d/ subjacentes. Os exemplos em (1), (2) e (3), abaixo, são todas ocorrências do corpus analisado em que houve aplicação da regra de HS:

(1)

Vontade de conhecer $>$ vonta de conhecer

Qualidade de vida $>$ qualida de vida

Monte de gente $>$ mon de gente

Acidente de trânsito $>$ aciden de trânsito

(2)

Resto do ano $>$ res do ano

Fundo do poço $>$ fun do poço

Desconhecimento total $>$ desconhecimen total

Dentro do consultório $>$ den do consultório

Elisa Battisti é professora do Departamento de Letras, Mestrado em Letras e Cultura Regional da Universidade de Caxias do Sul. 
(3)

Completamente diferente $>$ completamen diferente

Mercado de trabalho $>$ merca de trabalho

Universidade do México > Universida do México

O percentual de aplicação da regra foi de $21 \%$, em 284 de 1.341 contextos levantados. As variáveis independentes selecionadas foram lingüísticas: Qualidade das Vogais, Posição em Relação à Frase Fonológica ${ }^{4}$. A aplicação da regra foi favorecida em sílabas com a mesma vogal, dentro da frase fonológica. Em (1) e (2), acima, as vogais-núcleo (subjacentes) das sílabas envolvidas são idênticas, as consoantes podem diferir em sonoridade; em (3), vogais-núcleo subjacentes não são idênticas, e as consoantes diferem em sonoridade, mostrando mais claramente que o material fonológico que se realiza no output é o da sílaba da direita ${ }^{5}$. Tendo em conta esses fatos, como conceber haplologia? Como um processo de apagamento ou de coalescência? Que princípio(s) desencadearia(m)/ regeria $(\mathrm{m})$ o processo?

\section{CONCEPÇÕES TRADICIONAIS DE HS}

Haplologia sintática é assim definida por Silveira (1971), um dos poucos autores que registram esse processo variável do português brasileiro:

No terreno da fonética sintática o fato é, na essência, o mesmo: a sílaba

final de um vocábulo (ou mesmo um monossílabo) se encontra com um

monossílabo ou sílaba seguinte, foneticamente igual ou semelhante ao

monossílabo (ou sílaba) anterior, e um desses elementos (sílaba ou

monossílabo) desaparece na pronúncia. (Silveira 1971, p.79)

Nessa definição, predomina a informação sobre o contexto de aplicação, mais que sobre a natureza do processo, uma vez que o autor menciona apenas desaparecimento como resultado da regra.

Apagamento e degeminação como partes do processo de haplologia é proposta de Sá Nogueira (1938, 1941). Na descrição do autor, duas palavras em seqüência na frase cujas sílaba final e inicial, respectivamente, são idênticas ou muito similares, a vogal final da primeira é apagada, as duas consoantes tornam-se uma geminada, e elas finalmente reduzem a uma consoante. Apagamento da vogal e degeminação da consoante são, então, os dois processos envolvidos na haplologia sintática.

Seguindo-se essa interpretação, é apagamento o que leva à não-realização de material fonológico no output. Por que, então, questionar a natureza do processo, a possibilidade de coalescência estar em jogo? Mais que uma questão sobre os dados, essa é uma pergunta voltada à formalização da regra, origina-se das possibilidades (teóricas) de representar o processo através de distintas interações de restrições, e de diferentes restrições envolvidas na não-realização de estrutura. Na seção que segue, tais possibilidades são explicitadas pela revisão de de Lacy (1999) e parted a dissertação de doutorao de Gouskova (2003). Embora os autores abordem haplologia morfológica (HM), a revisão de suas propostas se faz necessária pela discussão acerca do papel desempenhado por restrições como o OCP - que, numa abordagem baseada em restrições como a TO, corresponde a uma demanda da gramática pela não-identidade de seqüências de segmentos - e pela ilustração de que, dependendo da interação de restrições através de diferentes ordenamentos na hierarquia, tanto candidatos que sofrem haplologia quanto candidatos que sofrem apagamento podem ser selecionados como a forma ótima.

\section{CONCEPÇÕES DE HAPLOLOGIA PELA TEORIA DA OTIMIDADE (TO)}

\subsection{Haplologia como economia (De Lacy, 1999)}

No francês, haplologia morfológica (HM) ocorre em derivações como deixis + ist $\rightarrow$ [deiksist], e não *[deiksisist], em que, de dois morfemas subjacentes fonologicamente idênticos, somente uma seqüência aparece na superfície. Conforme de Lacy (1999), HM é coalescência: o material fonológico dos dois morfemas se funde no output. $\mathrm{O}$ desencadeador da HM é qualquer restrição de marcação C relevante, isto é, qualquer restrição de marcação que é violada pelo morfema que sofre HM. Por exemplo, *coronal é relevante para $t a$ (pode desencadear a HM de $t a$ ), mas *labial não é. Essa proposta é formalizada em termos de TO através da hierarquia de restrições MAX $>>C>>$ UNIFORMITY. MAX requer que a 
todo segmento de input corresponda um segmento de output (proibido apagamento), e UNIFORMITY, que nenhum segmento de output corresponda a mais de um segmento de input (proibido coalescência). A hierarquia requer que segmentos subjacentes sejam preservados enquanto permite que eles coalesçam de modo a produzir um output com menos estrutura. Um exemplo (de Lacy 1999, p.3):

(4)

\begin{tabular}{|l|c|c|c|}
\hline$/ \mathrm{t}_{1} \mathrm{a}_{2} /+/ \mathrm{t}_{3} \mathrm{a}_{4} /$ & MAX & $\mathrm{C}$ & UNIFORMITY \\
\hline (a) $\mathrm{t}_{1} \mathrm{a}_{2} \mathrm{t}_{3} \mathrm{a}_{4}$ & & $\mathrm{x} !$ & \\
\hline (b) $\mathrm{t}_{1} \mathrm{a}_{2}$ & $\mathrm{xx} !$ & & \\
\hline (c) $\mathrm{t}_{1}, \mathrm{a}_{2}{ }_{2}$, & & & $\mathrm{xx}$ \\
\hline
\end{tabular}

Tanto MAX quanto UNIFORMITY são restrições de fidelidade: MAX penaliza o candidato (b), acima, porque o segundo morfema foi apagado; UNIFORMITY penaliza (c) porque houve coalescência, mas mesmo assim esse é escolhido como forma ótima porque não viola a restrição de marcação representada por $\mathrm{C}$, como faz o candidato (a), que é eliminado.

A hierarquia no tableau acima é a básica. Não está completa porque permite HM entre quaisquer dois afixos, sejam eles idênticos ou não. A identidade é reforçada pela restrição IDENT-F, que milita pela identidade de traço ao requerer valores de traço idênticos entre segmentos correspondentes no par de representação input-output. Segundo de Lacy, a coalescência de segmentos não-idênticos não ocorre porque isso fere IDENT-F. Observe-se (de Lacy 1999, p.5):

(5)

\begin{tabular}{|l|c|c|c|c|}
\hline$/ \mathrm{t}_{1} \mathrm{a}_{2} /+/ \mathrm{d}_{3} \mathrm{i}_{4} /$ & MAX & IDENT-F & $\mathrm{C}$ & UNIFORMITY \\
\hline \multirow{3}{*}{$(\mathrm{a}) \mathrm{t}_{1} \mathrm{a}_{2} \mathrm{~d}_{3} \mathrm{i}_{4}$} & & & $\mathrm{x} !$ & \\
\hline (b) $\mathrm{t}_{1} \mathrm{a}_{2}$ & $\mathrm{xx} !$ & & & \\
\hline (c) $\mathrm{t}_{1},{ }_{3} \mathrm{a}_{2},{ }_{4}$ & & $\mathrm{xx} !$ & & $\mathrm{xx}$ \\
\hline
\end{tabular}

O candidato (c), que sofre coalescência, supera (b), em que há apagamento, mas com IDENTF em posição alta na hierarquia, abaixo de MAX, a mudança de traço implicada na coalescência é penalizada, e a forma (a), sem apagamento ou coalescência, emerge como candidato ótimo.

Nesse ponto, poder-se-ia perguntar por que conceber HM como coalescência é melhor que concebê-la como apagamento. Conforme de Lacy
(1999), abordar não-realização de estrutura como apagamento ou coalescência significa fazer diferentes previsões sobre a afiliação morfológica de segmentos de output. $\mathrm{Na}$ abordagem de haplologia como apagamento, o material fonológico de um dos morfemas não está presente no output, enquanto na abordagem como coalescência ele é preservado. Prevê-se que restrições que se refiram a afiliação morfológica de um segmento apliquemse diferentemente nos dois casos.

O japonês fornece ilustração. Na afixação do sufixo predicativo - si [Si] a uma base como /kanasi/

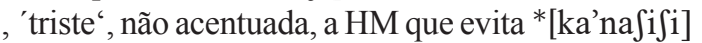
e produz [ka'nasi] pode ser concebida como apagamento de raiz, de sufixo ou como coalescência, mas cada abordagem fará uma previsão diferente sobre a posição do acento. A acentuação é fornecida, no caso do sufixo - si, pelo próprio sufixo: ele induz o acento a aparecer na sílaba que o precede imediatamente em bases não-acentuadas. Se concebida como apagamento de afixo, a forma viria à superfície sem acento *[kana $\mathrm{ji}$, ou se instanciaria um caso de opacidade, mesmo assim com o acento na posição errada: *[kana'ji]. Se concebida como apagamento de raiz, a posição do acento estaria correta em bases não-acentuadas como/kanasi/, mas errada em bases acentuadas, quando a acentuação por afixação de -si faz com que o acento caia na penúltima vogal da raiz (/siro/'branco' + -si $\rightarrow$ ['Sirofi] . Isso mostra que o [Si] que resulta da HM deve pertencer tanto à raiz quanto ao sufixo simultaneamente, o que sustenta a abordagem como coalescência.

Uma questão levantada por de Lacy diz respeito à restrição de marcação envolvida na haplologia, e pode assim ser formulada: por que qualquer restrição de marcação (relevante) é melhor que OCP como desencadeador da HM, se OCP milita contra identidade de seqüências de segmentos?

$\mathrm{O}$ autor denomina OCP-generalizado a restrição que diretamente bane seqüências idênticas de segmentos adjacentes, extensão do princípio de Leben (1973) e McCarthy (1986), o “OCP tradicional" . De Lacy (1999) afirma que o OCPgeneralizado pode produzir HM como apagamento, posicionando-se OCP e UNIFORMITY acima de MAX na hierarquia (ver o tableau em (6)). E pode produzir HM como coalescência, posicionando-se OCP e MAX acima de UNIFORMITY (ver o tableau em (7)): 
(6) OCP com apagamento

\begin{tabular}{|l|c|c|c|}
\hline$/ \mathrm{t}_{1} \mathrm{a}_{2} /+/ \mathrm{t}_{3} \mathrm{a}_{4} /$ & OCP( $t a)$ & UNIFORMITY & MAX-ta \\
\hline (a) $\mathrm{t}_{1} \mathrm{a}_{2} \mathrm{t}_{3} \mathrm{a}_{4}$ & $\mathrm{x} !$ & & \\
\hline$(\mathrm{b}) \mathrm{t}_{1} \mathrm{a}_{2}$ & & & $\mathrm{x}$ \\
\hline (c) $\mathrm{t}_{1}, \mathrm{a}_{3} \mathrm{a}_{2}{ }_{4}$ & & $\mathrm{xx} !$ & \\
\hline
\end{tabular}

\begin{tabular}{|l|c|c|c|}
\hline$/ \mathrm{t}_{1} \mathrm{a}_{2} /+/ \mathrm{d}_{3} \mathrm{i}_{4} /$ & OCP $(t a)$ & UNIFORMITY & MAX-ta \\
\hline (a) $\mathrm{t}_{1} \mathrm{a}_{2} \mathrm{~d}_{3} \mathrm{i}_{4}$ & & & \\
\hline (b) $\mathrm{t}_{1} \mathrm{a}_{2}$ & & & $\mathrm{x} !$ \\
\hline (c) $\mathrm{t}_{1,} \mathrm{a}_{2},{ }_{4}$ & & $\mathrm{xx} !$ & \\
\hline
\end{tabular}

(7) OCP com coalescência

\begin{tabular}{|l|c|c|c|}
\hline$/ \mathrm{t}_{1} \mathrm{a}_{2} /+/ \mathrm{t}_{3} \mathrm{a}_{4} /$ & OCP $(t a)$ & MAX- $t a$ & UNIFORMITY \\
\hline (a) $\mathrm{t}_{1} \mathrm{a}_{2} \mathrm{t}_{3} \mathrm{a}_{4}$ & $\mathrm{x} !$ & & \\
\hline (b) $\mathrm{t}_{1} \mathrm{a}_{2}$ & & $\mathrm{x} !$ & \\
\hline (c) $\mathrm{t}_{1,3} \mathrm{a}_{2}, 4$ & & & $\mathrm{xx}$ \\
\hline
\end{tabular}

\begin{tabular}{|c|c|c|c|}
\hline$/ \mathrm{t}_{1} \mathrm{a}_{2} /+/ \mathrm{d}_{3} \mathrm{i}_{4} /$ & $\mathrm{OCP}(t a)$ & MAX-ta & UNIFORMITY \\
\hline$\checkmark$ (a) $t_{1} a_{2} d_{3} i_{4}$ & & & \\
\hline (b) $t_{1} a_{2}$ & & $\mathrm{x}$ ! & \\
\hline (c) $\mathrm{t}_{1,3} \mathrm{a}_{2}, 4$ & & & $\mathrm{xx}$ ! \\
\hline
\end{tabular}

A restrição OCP-generalizado em posição alta na hierarquia produz $\mathrm{HM}$ ao penalizar candidatos com conjuntos de elementos (sílabas) adjacentes idênticos. HM como coalescência ou apagamento resulta da posição de UNIFORMITY em relação a MAX. OCP generalizado seria, então, a restrição $C$ relevante envolvida na haplologia, como defende de Lacy. É aí que o autor salienta um aspecto decorrente do processo, a economia de estrutura, relativamente à questão da identidade.

De Lacy reforça que a HM apresenta dois aspectos: é uma tentativa de minimizar estrutura (e, mais geralmente, evitar violação a restrições) e ocorrerá somente se todas as especificações de traço subjacentes se mantiverem no output (exigência de identidade). $\mathrm{Na}$ abordagem de haplologia como coalescência, pela qual de Lacy advoga, identidade é assegurada por IDENT-F e minimização de estrutura deve-se à restrição de marcação desencadeadora. $\mathrm{Ou}$ seja, essas duas demandas correspondem, cada qual, a uma restrição distinta. $\mathrm{Na}$ abordagem com OCP, amalgamam-se as duas demandas em uma restrição que permite HM como apagamento. De Lacy defende que uma restrição como OCP-generalizado, que bane seqüências idênticas de segmentos, é indesejável e desnecessária, estabelece uma relação de segunda ordem (entre seqüências, não entre elementos), constituindo uma adição à gramática.
Além disso, versões do OCP-generalizado, como a de Yip (1998) e Plag (1998), que propõem exigência de não-identidade também entre constituintes, o primeiro, entre morfológicos e fonológicos, o segundo, entre fonológicos apenas, torna OCP muito complexo, pois tem que dar conta de casos que não ocorrem entre afixos, mas entre afixo e radical, por exemplo. "Em todo o caso, mesmo que o OCPtradicional seja mantido, haplologia como apagamento só é possível com afixos monosegmentais; em todos os outros casos, deve ser coalescência." (de Lacy 1999, p.18).

Não há, portanto, uma restrição específica para HM (como seria o OCP-generalizado). Qualquer restrição de marcação é desencadeador potencial, desde que violada mais na forma nãohaplologizada que na forma haplologizada. De Lacy (1999) afirma que *STRUC (no phonological structure) seria essa restrição de marcação ideal, pois a forma haplologizada sempre terá menos estrutura que a não-haplologizada.

Pelo que se viu até aqui da proposta de de Lacy (1999), dois pontos é necessário abordar em uma interpretação de haplologia pela TO: a natureza do processo, se apagamento ou coalescência (e, como exposto acima, de Lacy sustenta coalescência para $\mathrm{HM}$ ) e a restrição de marcação em jogo. No que tange à haplologia sintática, de interesse no presente trabalho para dados do português brasileiro, veremos adiante que OCP poderia ser essa restrição de marcação, mas alguns problemas decorreriam de uma tal interpretação. Antes de passarmos a esse ponto, uma última questão surge da proposta de de Lacy (1999) para HM, e passará a ser abordada: como dar conta de HM com identidade parcial?

Entre os exemplos que de Lacy (1999) fornece de HM com identidade parcial está o do francês /analiz/ analyse $+/$ ist $/ \rightarrow$ [analist], e não *[analizist]. O autor diz que "alguns traços simplesmente não importam na haplologia com identidade parcial.[...] em Francês, o traço [voz] é irrelevante ao computar-se a identidade de seqüências adjacentes..." (de Lacy 1999, p.22). Isso significa que IDENT[voz] deve ser ranqueado mais baixo na hierarquia, abaixo da restrição (de marcação) desencadeadora da HM, o que implica que é mais harmônico instanciar-se a HM e ser infiel à especificação [voz] subjacente, que violar a restrição de marcação. Considerando-se que IDENTF' representa o conjunto de restrições IDENT que se 
refere a qualquer traço subjacente exceto [voz], observe-se o que seria a avaliação da forma do francês:

(8)

\begin{tabular}{|c|c|c|c|c|c|}
\hline /anali $\mathrm{z}_{2} /++\mathrm{i}_{3} \mathrm{~s}_{4} t /$ & MAX & IDENT-F' & *Struc & IDENT[VoZ] & UNIFORMITY \\
\hline (a) anali $s_{2}, t$ & $\mathrm{xx!}$ & & & & \\
\hline (b) analizizi $i_{2} \mathrm{~s}_{4} \mathrm{t}$ & & & $\mathrm{xx} !$ & & \\
\hline F(c) anali ${ }_{1,3} \mathrm{~S}_{2,4} \mathrm{t}$ & & & & $\mathrm{x}$ & $\mathrm{xx}$ \\
\hline
\end{tabular}

(de Lacy 1999, p.22)

Como se vê no tableau acima, candidatos que preservam estrutura ((b) e (c)) são sempre melhores que aquele que apaga estrutura ((a)) se MAX está no topo da hierarquia. O candidato (c) é a forma ótima, apesar da não-identidade de vozeamento, porque IDENT[voz] é desmembrado da restrição IDENT-F' e dominado por *Struc. Assim, mantém-se a hierarquia com IDENT-F' junto a MAX em posição alta na hierarquia, e UNIFORMITY em posição baixa, fazendo emergir a forma com coalescência no output.

A interpretação de de Lacy (1999) de HM com identidade parcial parece adequada à haplologia sintática no português brasileiro, em que as consoantes no onset das sílabas envolvidas podem diferir em vozeamento. O exercício de aplicação dessa interpretação aos dados deste estudo, no entanto, virá após avaliar-se a concepção de uma gramática com ${ }^{*}$ STRUC, como adota de Lacy. É o que se fará na seção seguinte, revisandose a hipótese de Gouskova (2003) para o processo de síncope que é a HM.

\subsection{Haplologia como efeito de economia (Gouskova, 2003)}

Gouskova (2003) sustenta que as restrições de marcação na TO são lenientes: uma forma só pode ser marcada se houver uma forma não-marcada na língua. Por exemplo, num sistema em que vogais nasais são marcadas, as orais não são, o que significa que há uma restrição * NASALV em Con (que é finito), mas não há uma restrição contra vogais orais ou todas as vogais. “... as restrições são limitadas naquilo que elas podem banir; a idéia de que 'tudo é marcado` é expressamente proibida." (Gouskova 2003, p.2). A conseqüência dessa idéia é que não há restrições de economia que penalizem estrutura, para as quais uma forma com menos estrutura é mais harmônica do que uma com mais estrutura, como
*STRUC prevê. Se assim fosse, zero ou nenhuma estrutura seria sempre melhor que as formas lingüísticas que se realizam.

Todas as restrições de marcação são derivadas de escalas de harmonia, mas nem todos os níveis de uma escala possuem uma restrição de marcação correspondente. Para o exemplo das vogais nasais, a autora propõe a escala vogal oral mais harmônico que vogal nasal. Nenhuma escala implica que zero seja melhor que estrutura. Isso é um princípio na proposta de Gouskova. As escalas podem expressar marcação de uma estrutura relativamente a uma outra, mas não podem expressar economia. Efeitos de economia derivam da interação de restrições de marcação lenientes.

Haplologia morfológica é um caso de apagamento de estrutura de input, pela autora concebido como um efeito de economia. Esse apagamento "envolve segmentos idênticos adjacentes (efeitos de OCP) ou seqüências (haplologia)." (Gouskova 2003, p.55). O processo corresponde à não-realização de um morfema quando ele é anexado a um radical que contém uma seqüência de fonemas adjacentes idênticos, como no caso do sufixo -iste do francês. Gouskova afirma que a haplologia deve ser discutida no contexto de $\mathrm{OCP}$, restrição bastante geral contra identidade que é satisfeita de várias formas: dissimilação, alomorfia, gaps lexicais, apagamento de consoante, síncope. A autora acredita que haplologia seja mais uma dessas formas, previstas pela interação de OCP com MAX.

Gouskova (2003) é contra o modo como de Lacy (1999) sustenta a concepção de HM como coalescência, uma concepção em que o processo resulta das exigências de uma família de restrições de economia representada por *StRuc. A autora é contra especialmente o ataque que de Lacy (1999) faz a OCP, em defesa a *STruc. Segundo ela, "somente OCP pode ser satisfeito por dissimilação, alomorfia e outros processos que não envolvem apagamento ou coalescência." (Gouskova 2003, p.57). Gouskova defende que todos os efeitos de economia podem e deveriam ser analisados em termos de restrições de marcação (lenientes, na proposta dela).

Até aqui, então, contrastando-se a proposta de de Lacy (1999) e a hipótese de Gouskova (2003), mais que a natureza do processo de HM, o que se discute é a restrição de marcação que é satisfeita com a não-realização de estrutura. Pensou-se que, para se chegar a essa restrição no que tange à 
haplologia sintática (HS) e ao português brasileiro, fosse esclarecedora uma aproximação da HS com outros processos de sândi externo. É o que se fará a seguir, revisando-se brevemente a interpretação de Bisol (1996, 2002, 2003) para o sândi vocálico externo.

\section{HAPLOLOGIA SINTÁTICA E O SÂNDI VOCÁLICO EXTERNO}

O que há em comum entre HS e processos de sândi vocálico externo que resultam na nãorealização de material fonológico? Que princípio estaria por trás desses processos de sândi externo, o vocálico e a HS? Essas são questões que a revisão dos estudos de Bisol sobre o sândi vocálico externo no português brasileiro pode auxiliar a responder.

Ditongação (carro aberto > carrwabertu), elisão (camisa usada > camisusada) e degeminação (camisa azul > camisazul) são processos de sândi vocálico externo passíveis de ocorrer no nível da frase. Os três processos são abordados em Bisol (1996); elisão e degeminação, em Bisol (2002, 2003). Embora os tenha elaborado com objetivos diferentes, em todos esses trabalhos a autora aponta o choque de duas rimas como o desencadeador do sândi vocálico externo e a ressilabação, ativada por um princípio lingüístico ${ }^{7}$, o que dirige todo o processo. Em jogo está também uma restrição rítmica a que se sujeitam a elisão e a degeminação: "não se aplicam ou fazem-se raras se a segunda vogal for portadora do acento principal da frase" (Bisol, 2002, p.249); “a ditongação, por outro lado, não é bloqueada nesse ambiente e ocorre sempre que a vogal alta numa seqüência VV for átona" (Bisol 2003, p.177).

No processo de sândi que é a HS, o choque de rimas não é o gatilho da regra, uma vez que a seqüência envolvida é de duas sílabas CV; o desencadeador do processo não é o que há em comum entre haplologia e ditongação, elisão, degeminação; o que há em comum é o resultado da regra. Especificamente no que se refere aos processos de elisão e degeminação, esse resultado em comum é a não-realização de material fonológico, predominantemente átono. No sândi vocálico, a estrutura apagada é a da sílaba à esquerda, como seria na HS. Apagada a estrutura silábica, no contexto de elisão a vogal flutuante (não-licenciada) é também apagada; no contexto de degeminação, a vogal flutuante é reassociada à vogal da sílaba subsistente em função do OCP, e a vogal longa resultante é posteriormente reduzida.

O OCP tem papel na degeminação e, como Bisol (1996, p.167) refere, versões anteriores de seu estudo apresentavam OCP, e não choque silábico, como o princípio desencadeador do processo. Portanto, considerando-se outros processos de sândi externo como o vocálico, poder-se-ia admitir que OCP tivesse papel frente à HS. A versão do OCP envolvida seria OCPgeneralizado, não o OCP tradicional (ver seção 3.1, acima). Isso implicaria desconsiderar as críticas de de Lacy (1999) a OCP, por esse autor visto (a) como uma restrição com papel duplo, o de militar contra identidade de elementos em seqüência e também o de requerer minimização de estrutura; (b) como uma restrição que estabelece uma relação de segunda ordem (entre seqüências, não entre elementos), permitindo apagamento. No entanto, assumindo-se OCP como a restrição de marcação, estar-se-ia evitando conceber a existência em CON (e na gramática) de uma restrição que preferisse a realização de nenhuma estrutura a qualquer estrutura, como na proposta de de Lacy (1999) a restrição de marcação *STRUC expressa, crítica essa feita por Gouskova, como se viu (seção 3.2).

Assim, da revisão de Bisol (1996, 2002, 2003) e dos trabalhos de de Lacy (1999) e Gouskova (2003) na linha da TO, pode-se tomar OCP como a restrição que demanda não-realização de estrutura no contexto de identidade de elementos em seqüência, na palavra ou na frase, ambiente de haplologia. Isso significa assumir que, apesar de na análise de regra variável termos selecionado apenas seqüências com/t/ e /d/ subjacentes, também sílabas contíguas com segmentos (idênticos ou parcialmente idênticos) possam ser afetadas por $\mathrm{HS}^{8}$.

Abordada a questão da restrição de marcação envolvida na HS, resta a da natureza do processo, se apagamento ou coalescência. É o que se discutirá na seção seguinte, procurando-se interpretar a HS pela Teoria da Otimidade (TO $)^{9}$.

\section{HAPLOLOGIA SINTÁTICA PELA TO}

Diferentemente dos casos de HM referidos 
nos estudos, em que o processo é categórico, a HS no português brasileiro é regra variável. Atinge seqüências idênticas de segmentos e seqüências parcialmente idênticas, em que o traço [voz] da consoante é irrelevante, como exemplificam as ocorrências em (1) e (2); e às vezes em que tanto o traço [voz] quanto a qualidade das vogais pode ser diferente, como nos exemplos em (3).

Como vimos, embora de Lacy (1999) advogue por uma análise de HM como coalescência e sem OCP, afirma que uma análise com essa restrição pode levar tanto a apagamento como a coalescência. Assim, testaram-se ambas as interpretações de HS com OCP conforme de Lacy (1999), em que OCP interage com MAX e UNIFORMITY em diferentes posições na hierarquia para seqüências idênticas de segmentos e para seqüências parcialmente idênticas de segmentos. Como a análise de regra variável realizada levou em conta apenas seqüências de sílaba com $/ \mathrm{t} / \mathrm{e} / \mathrm{d} /$, tanto OCP quanto MAX não aparecem com contexto definido. Os tableaux de avaliação estão em (9), (10) e (11), abaixo. O resultado interessante é que em (9) e (10), a análise de HS em seqüências parcial ou totalmente idênticas dá certo concebendo-se a regra tanto como coalescência quanto como apagamento; já em (11), com OCP na hierarquia básica de de Lacy (1999) na posição da restrição de marcação, entre MAX e UNIFORMITY, a análise só dá certo (como coalescência) com seqüências totalmente idênticas. Em seqüências parcialmente idênticas, a forma não-haplologizada seria a mais harmônica, a não ser que se desmembrasse IDENT-F' de IDENT[traço específico], como de Lacy (1999) faz (ver (h) acima), o que de alguma forma torna sua proposta menos geral. Está-se diante de uma razão (formal) reforçando HS como apagamento e não como coalescência.

(9) HS: OCP com apagamento

\begin{tabular}{|l|l|l|l|}
\hline$/$ kwalidad $\mathrm{e}_{2} / / \mathrm{d}_{3} \mathrm{e}_{4} / /$ vida/ & OCP & UnIFORMITY & MAX \\
\hline (a) $\mathrm{d}_{1} \mathrm{e}_{2} \mathrm{~d}_{3} \mathrm{e}_{4}$ & $\mathrm{xx} !$ & & \\
\hline (b) $\mathrm{d}_{3} \mathrm{e}_{4}$ & & & $\mathrm{xx}$ \\
\hline (c) $\mathrm{d}_{1{ }^{3}} \mathrm{e}_{2}{ }_{2}$ & & $\mathrm{xx} !$ & \\
\hline
\end{tabular}

\begin{tabular}{|l|l|c|c|}
\hline$/ \mathrm{moNt}_{1} \mathrm{e}_{2} / / \mathrm{d}_{3} \mathrm{e}_{4} / / \mathrm{ZeNte}^{\mathrm{e}} /$ & OCP & UNIFORMITY & MAX, IDENT \\
\hline (a) $\mathrm{t}_{1} \mathrm{e}_{2} \mathrm{~d}_{3} \mathrm{e}_{4}$ & $\mathrm{x} !$ & & \\
\hline (b) $\mathrm{d}_{3} \mathrm{e}_{4}$ & & & $\mathrm{xx}$ \\
\hline (c) $\mathrm{d}_{1,3} \mathrm{e}_{2}{ }_{4}$ & & $\mathrm{xx} !$ & $\mathrm{X}$ \\
\hline
\end{tabular}

(10) HS: OCP com coalescência

\begin{tabular}{|l|c|c|c|}
\hline fuNd $\mathrm{d}_{1} / / \mathrm{d}_{3} \mathrm{o}_{4} / /$ poso/ & OCP & MAX & UNIFORMITY \\
\hline (a) $\mathrm{d}_{1} \mathrm{o}_{2} \mathrm{~d}_{3} \mathrm{o}_{4}$ & $\mathrm{xx} !$ & & \\
\hline (b) $\mathrm{d}_{1} \mathrm{o}_{2}$ & & $\mathrm{xx} !$ & \\
\hline (c) $\mathrm{d}_{1,3} \mathrm{o}_{2}, 4$ & & & $\mathrm{xx}$ \\
\hline
\end{tabular}

\begin{tabular}{|l|c|c|c|}
\hline$/$ rest $_{1} \mathrm{o}_{2} / / \mathrm{d}_{3} \mathrm{o}_{4} / / \mathrm{ano} /$ & OCP & MAX, IDENT & UNIFORMITY \\
\hline (a) $\mathrm{t}_{1} \mathrm{o}_{2} \mathrm{~d}_{3} \mathrm{o}_{4}$ & $\mathrm{x} !$ & & \\
\hline (b) $\mathrm{d}_{3} \mathrm{o}_{4}$ & & $\mathrm{xx} !$ & \\
\hline (c) $\mathrm{d}_{1}{ }^{\circ}{ }_{3}{ }^{\circ}{ }^{\prime}$ & & $\mathrm{x} !$ & $\mathrm{xx}$ \\
\hline
\end{tabular}

(11) HS: OCP na hierarquia básica de de Lacy (1999)

\begin{tabular}{|c|c|c|c|}
\hline$/$ kwalidad $_{1} \mathrm{e}_{2} / / \mathrm{d}_{3} \mathrm{e}_{4} / / \mathrm{vida} /$ & $\overline{M A X}$ & $\overline{\mathrm{OCP}}$ & $\overline{\text { UNIFORMITY }}$ \\
\hline (a) $\mathrm{d}_{1} \mathrm{e}_{2} \mathrm{~d}_{3} \mathrm{e}_{4}$ & & $\mathrm{xx}$ ! & \\
\hline (b) $\mathrm{d}_{3} \mathrm{e}_{4}$ & $\mathrm{xx} !$ & & \\
\hline (c) $\mathrm{d}_{1 \gamma_{3}} \mathrm{e}_{2,4}$ & & & $\mathrm{xx}$ \\
\hline
\end{tabular}

\begin{tabular}{|l|c|c|c|}
\hline$/$ fuNd $_{1} \mathrm{O}_{2} / / \mathrm{d}_{3} \mathrm{O}_{4} / /$ poso/ & MAX & OCP & UNIFORMITY \\
\hline (a) $\mathrm{d}_{1} \mathrm{o}_{2} \mathrm{~d}_{3} \mathrm{o}_{4}$ & & $\mathrm{xx} !$ & \\
\hline (b) $\mathrm{d}_{1} \mathrm{o}_{2}$ & $\mathrm{xx} !$ & & \\
\hline (c) $\mathrm{d}_{1,3} \mathrm{o}_{2},{ }_{4}$ & & & $\mathrm{xx}$ \\
\hline
\end{tabular}

\begin{tabular}{|l|c|c|c|}
\hline$/ \mathrm{moNt}_{1} \mathrm{e}_{2} / / \mathrm{d}_{3} \mathrm{e}_{4} / / 3 \mathrm{eNte} /$ & MAX, IDENT & OCP & UNIFORMITY \\
\hline$\widetilde{N}(\mathrm{a}) \mathrm{t}_{1} \mathrm{e}_{2} \mathrm{~d}_{3} \mathrm{e}_{4}$ & & $\mathrm{x}$ & \\
\hline (b) $\mathrm{d}_{3} \mathrm{e}_{4}$ & $\mathrm{xx} !$ & & \\
\hline (c) $\mathrm{d}_{1}, \mathrm{e}_{2} \mathrm{e}_{24}$ & $\mathrm{x} !$ & & $\mathrm{xx}$ \\
\hline
\end{tabular}

\begin{tabular}{|l|c|l|c|}
\hline$/$ rest $\mathrm{O}_{2} / / \mathrm{d}_{3} \mathrm{O}_{4} / /$ ano/ & MAX, IDENT & OCP & UNIFORMITY \\
\hline$\%$ (a) $\mathrm{t}_{1} \mathrm{O}_{2} \mathrm{~d}_{3} \mathrm{O}_{4}$ & & $\mathrm{x}$ & \\
\hline (b) $\mathrm{d}_{3} \mathrm{O}_{4}$ & $\mathrm{xx} !$ & & \\
\hline (c) $\mathrm{d}_{1^{\prime}{ }^{\prime}{ }_{2}{ }^{\prime}}$ & $\mathrm{x} !$ & & $\mathrm{xx}$ \\
\hline
\end{tabular}

\section{CONSIDERAÇÕES FINAIS}

Em estudos como os de Sá Nogueira (1938, 1941) e Silveira (1971), a HS do português é referida como apagamento. A necessidade de discutir a hipótese de HS como coalescência foi motivada pelo aparato formal da TO e trabalhos recentes envolvendo a HM realizados nessa perspectiva. Abusca de características comuns entre a HS e outros processos de sândi permitiu conceber OCP como o desencadeador da HS. Contrastando-se a análise pela TO com OCP (em posição alta na hierarquia) como apagamento e coalescência, não se verifica superioridade de uma e outra versão de haplologia; nelas OCP necessita ser satisfeito à custa de fidelidade. $O$ desempate pela versão de HS como apagamento no português brasileiro vem da análise com OCP abaixo de MAX, conforme a 
Hierarquia Básica de de Lacy (1999). Por ela, só seqüências idênticas de segmentos sofrem haplologia (por coalescência); a seqüências parcialmente idênticas, como as que podem sofrer HS no português brasileiro, o processo não se aplica. Dessa análise, conclui-se que na seqüência (sintática) de sílabas com /t/ e /d/ subjacentes, a identidade dos segmentos é o que desencadeia a HS, quando a fidelidade é desrespeitada. As formas não-haplologizadas, com as quais as haplologizadas alternam no português brasileiro, são fiéis.

\section{REFERÊNCIAS BIBLIOGRÁFICAS}

BISOL, L. O sândi e a ressilabação. Letras de Hoje. Porto Alegre. v.31, n.2, p.159-168, junho de 1996. . A degeminação e a elisão no VARSUL. In: BISOL, L. e BRESCANCINI, C. (orgs.) Fonologia e variação: recortes do português brasileiro. PortoAlegre: EDIPUCRS, 2002.

Sandhi in Brazilian Portuguese. Probus 15. p.177-200, 2003.

De LACY, P. Morphological haplology and correspondence. In: De LACY, P. \& NOWAK, A. (eds.) University of Massachusetts Occasional Papers: Papers form the $25^{\text {th }}$ Reunion. Amherst, MA: GLSA, 1999. (ROA 289).

GOUSKOVA, M. Deriving economy: syncope in Optimality Theory. University of Massachusetts, Amherst: Ph.D. Dissertation, 2003.

ITÔ, J. Syllable theory in prosodic phonology. MIT, Amherst:Ph.D. Dissertation, 1986.

LEBEN, W. Suprasegmental Phonology.MIT: Ph.D. Dissertation, 1973.

McCARTHY, J. OCP effects: gemination and antigemination. Linguistic Inquiry 17, 1986. p.207-263. McCARTHY, J. \& PRINCE, A. Prosodic morphology $I$ - constraint interaction and satisfaction. (não-publicado). Amherst/New Brunswick, Universidade de Massachusetts e Universidade de Rutgers, 1993.

Faithfulness and reduplicative identity. In: BECKMAN,J.N et al. (eds.) Papers in Optimality Theory - Umass Occasional Papers 18. Amherst, Massachusetts: GLSA,1995. p.249-384.

PLAG, Ingo. Morphological haplology in a constraint-based morpho-phonology. In: KEHREIN, W; WIESE, R. (Eds.) Phonology and morphology of the Germanic Languages. Tübingen: Niemeyer, 1998. p.199-215.
PRINCE,A. \& SMOLENSKY,P. Optimality theory: constraint interaction in generative grammar.(nãopublicado).NewBrunswick/Boulder: Universidade de Rutgers e Universidade do Colorado, 1993.

SÁ NOGUEIRA, R. Elementos para um tratado de fonética portuguesa. Lisboa: Centro de Estudos Filológicos, 1938.

- Tentativa de explicação dos fenômenos fonéticos do português. Lisboa: Livraria Clássica Editora, 1941.

SILVEIRA, S. Fonética sintática. Rio de Janeiro: Fundação Getúlio Vargas, 1971.

TENANI, L. E. Domínios prosódicos no português do Brasil: implicações para a prosódia e para a aplicação de processos fonológicos. Tese (Doutorado em Lingüística ) - Universidade Estadual de Campinas, 2002.

YIP, M. Identity avoidance in phonology and morphology. In. LAPOINTE, G. et.al. (Eds.) Morphology and its relation to phonology and syntax. Stanford, CA: CSLI Publications, 1998.p.216-246.

\section{Notas:}

${ }^{1}$ Uma versão preliminar deste trabalho foi apresentada no GT de Fonética e Fonologia da ANPOLL durante o XIX ENANPOLL, realizado em Maceió, de 28/06/2004 a 02/07/2004. ${ }^{2}$ Haplologia no Português do Sul do Brasil:Porto Alegre, análise em andamento, realizada junto ao grupo de pesquisa (CNPq) Aspectos fonológicos do português: sincronia e diacronia, liderado pela profa. Dra. Leda Bisol (PUCRS).

${ }^{3}$ Crystal (2000, p.49) define coalescência como "a união de unidades lingüísticas que antes podiam ser distinguidas", apontando fusão e merger como termos análogos.

${ }^{4} \mathrm{Na}$ variável Qualidade das Vogais, controlaram-se os fatores Sílabas com Mesma Vogal, Sílabas com Diferente Vogal; na variável Posição em Relação à Frase Fonológica, os fatores Dentro da Frase Fonológica, Entre Frases Fonológicas. Controlou-se o vozeamento das consoantes através da variável Sonoridade das Consoantes de Ataque, se idêntico ou diferente, mas a variável não foi selecionada.

${ }^{5}$ Tenani (2002) controlou o acento das sílabas em questão, entre frases fonológicas, e verificou que a haplologia é bloqueada apenas quando a primeira sílaba da seqüência é acentuada ( $D i \underline{i d}$

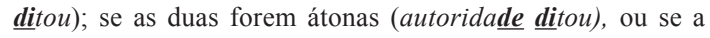
segunda for tônica e a primeira, átona (autorida $\underline{\text { de }} \underline{\text { dita }}$ ), o processo pode ocorrer.

${ }^{6}$ De Lacy explica que o OCP tradicional requer uma relação de não-identidade entre entidades (elementos individuais) adjacentes; o OCP generalizado, entre conjuntos de elementos. ${ }^{7}$ O Princípio de Licenciamento Prosódico, de Itô (1986), que requer que todas as unidades fonológicas sejam licenciadas prosodicamente. 
${ }^{8}$ Inicialmente incluídas no corpus e depois excluídas da análise de regra variável por sua quase raridade, ocorrências como compra pra mim > com pra mim podem-se verificar na fala do sul do Brasil.

${ }^{9}$ A presente análise dá conta dos resultados do estudo de regra variável referentes ao que se denominou contexto segmental de aplicação: qualidade das Cs e Vs envolvidas; não dá conta do contexto prosódico de aplicação, isto é, a posição em relação à frase fonológica, que se mostrou estatisticamente significativo. 